\title{
Carreira sem Fronteiras: Limite e Aplicabilidade de uma Teoria Clássica
}

\author{
Luciano Espósito Sewaybricker \\ Centro Universitário São Camilo, SP, Brasil.
}

\begin{abstract}
Resumo: Dentre as diversas teorias que se referem às atuais configurações dacarreira profissional, a da "carreira sem fronteiras" é uma das que detêm maior credibilidade nos meios acadêmico e empresarial. Contudo, quando se confrontam fundamentações teóricas com dados empíricos, percebe-se inconsistência. O objetivo deste artigo foi analisar essa inconsistência, através do conceito de "performatividade" apresentado pela Actor-Network Theory. Dessa forma, pode-se avaliar a "carreira sem fronteiras" não através de sua correspondência com a realidade, mas de sua reputação e influência no próprio cenário em que atua e, sobretudo, de sua eficiência em auxiliar as pessoas nas diversas ponderações que fazem em relação ao trabalho. Como resultados, identificou-se que a simplificação teórica favorece a reprodução da "carreira sem fronteiras"; que o modelo teórico corresponde à realidade de uma parcela pequena, mas altamente influente de profissionais dentro do universo de trabalho; e que há consonância entre o discurso da "carreira sem fronteiras" e o discurso gerencial típico das organizações flexíveis.
\end{abstract}

Palavras-chave: Trabalho, Mercado de Trabalho, Carreira sem Fronteiras.

\section{Boundaryless Career: Limitations and Applicability of a Classical Theory}

\begin{abstract}
Among the various theories that refer to the current settings of careers, the "boundaryless career" is one that holds more credibility both in academia and in business environment. However, there is a perceived inconsistency when confronting its theoretical predictions with empirical data. The aim of this study was to analyze this inconsistency through the concept of "performativity" presented by the Actor-Network Theory (ANT). Thus, one can evaluate the "boundaryless career' not through its correspondence with reality, but through its reputation and influence, and above all in its efficiency in assisting people in their evaluations of work related issues. The main results can be summarized as: the theoretical simplification of the context potentiate the "boundaryless career" reproduction; theory corresponds to the reality of a small portion, but highly influential professionals within the work context; there's an accordance between theory and the management discourse, leveraging performativity by flexible organizations.
\end{abstract}

Keywords: Work, Labor Market, Boundaryless Career. 


\title{
Carrera sin Fronteras: Límites y Aplicabilidad de una Teoría Clásica
}

\begin{abstract}
Resumen: Entre las muchas teorías que se refieren a los valores actuales de la carrera, la "carrera sin fronteras" es aquella que posee una mayor credibilidad en el mundo académico y de negocios. Sin embargo, cuando se enfrenta a sus predicciones teóricas con datos empíricos, se percibe inconsistencia. El objetivo de este estudio fue analizar esta incoherencia, a través del concepto de "performatividad", presentado por la teoría del actor-red (ANT). De esta manera, se puede evaluar la "carrera sin fronteras" no a través de su correspondencia con la realidad, pero su reputación e influencia en el escenario real en el que opera y, sobre todo, su eficiencia en ayudar a las personas en las diversas evaluaciones que hacen en relación al trabajo. Como resultado, se encontró que la simplificación teórica favorece la reproducción de la "carrera sin fronteras"; el modelo teórico se corresponde con la realidad de una pequeña parte, pero los profesionales altamente influyentes en el mundo del trabajo; y que no hay consonancia entre el discurso de la "carrera sin fronteras" y el discurso de la gente de gestión típica de las organizaciones flexibles.
\end{abstract}

Palabras clave: Trabajo, Mercado de Trabajo, Carrera sin Fronteras.

\section{Introdução}

\section{Carreira sem fronteiras?}

Na literatura de diversas áreas acadêmicas que tratam do tema trabalho, seja na Psicologia, na sociologia ou na administração, é muito comum encontrarmos referências às transformações pelas quais essa importante esfera da ação humana tem passado. Tais referências ganharam força após a década de 1970 em decorrência das novas tecnologias que passaram a fazer parte do cotidiano do trabalho (Davis, 2010; Malvezzi, 1999).

As novas tecnologias de comunicação e transporte permitiram que o mundo dos negócios se "desprendesse" do solo. Em um primeiro momento, os produtos e as informações passaram a transitar em fluxos globais; em um segundo momento, as próprias organizações tornaram-se fluidas e sem uma nacionalidade clara (Bauman, 2003). Tais fenômenos intensificaram, entre outras coisas, a velocidade com que as empresas passaram a se transformar para atender as novas e cada vez mais efêmeras demandas contemporâneas. A flexibilidade tornou-se desejada e necessária (Davis, 2010).

De um modo geral, essa transição de um cenário em que as grandes organizações eram estáveis e símbolo da economia (Davis, 2010) para outro, mais fluido e incerto (Bauman, 2003) gerou profundas mudanças na configuração do trabalho e nos estudos sobre a carreira. Essa transformação das grandes organizações abriu terreno para diversas teorias que apresentaram a nova realidade do trabalho e as perspectivas para a construção da carreira.

Dentre essas diversas teorias, a de "carreira sem fronteiras" (Arthur, 1994; Arthur, \& Rousseau, 1996) foi uma das que mais ganhou fama, provavelmente pela consonância com o zeitgeist da globalização, conforme Gunz, Evans e Jalland (2000) e Arnold e Cohen (2008) indicam. Segundo esses mesmos autores, a metáfora de se estar "sem fronteiras" mostrou-se hábil em traduzir a nova realidade das zonas de livre comércio e a da multinacionalização das empresas. Tal sucesso da teoria de "carreira sem fronteiras" foi tamanho, tanto no meio acadêmico quanto empresarial internacional e nacional, que se tornou uma espécie de status quo no estudo do tema "carreira" (Briscoe, Hall, \& Frautschy Demuth, 2006). Expressões como "o contexto de carreira sem fronteiras" (Higgins, Dobrow, \& Roloff, 2010, p. 749) e "a era da carreira sem fronteiras" (Eby, Butts, \& Lockwood, 2003, p. 689) são exemplos da proeminência dessa teoria (Inkson, Gunz, Ganesh, \& Roper, 2012).

No entendimento de Arthur (2008), corroborado por diversos outros autores, a crescente flexibilização da economia gerou uma diluição das estruturas do emprego. Se antes as grandes e estáveis organizações eram famosas por proporcionar emprego quase vitalício e progressão por tempo de serviço, diante do novo cenário foi preciso "afrouxar" as relações de trabalho e abrir espaço para adaptações mais ágeis. No fim dos 
anos 1980, a maioria das grandes organizações e dois terços das pequenas já haviam eliminado os planos de saúde nos Estados Unidos, antigo símbolo do compromisso de longo prazo (Davis, 2010).

A "carreira sem fronteiras", apresentada pela primeira vez por Arthur em 1994, seria o próprio antônimo da "carreira organizacional" citada acima. Segundo o autor: "Em linhas gerais, o cenário do emprego estável e da carreira organizacional está sumindo" (1994, p. 297, tradução nossa). Assim, na medida em que as organizações estariam menos dispostas a proporcionar estabilidade e progressão profissional, a carreira tornar-se-ia objeto próprio dos indivíduos (Dany, 2008). Não à toa é comum observar o crescente número de serviços de orientação de carreira e coaching que se propõem a auxiliar pessoas a se conectarem com suas carreiras pelo desenvolvimento de seu "propósito" ou "missão" pessoal. A própria definição de "carreira” para Arthur (Arthur, \& Hall, 1989, p. 8) traduz esse novo cenário: carreira é "a sequência evolutiva de experiências de trabalho de uma pessoa ao longo do tempo". Nessa concepção, "carreira" engloba uma perspectiva temporal, possivelmente fragmentada e pertencente ao indivíduo (Hall, 2002).

Outro aspecto central para a teoria da "carreira sem fronteiras” é, como o próprio nome já diz, a superação das diversas fronteiras existentes no universo do trabalho. Sejam fronteiras concretas, como as existentes entre organizações/empregadores, entre países e entre profissões, ou as fronteiras percebidas subjetivamente, todas elas estariam em processo de enfraquecimento diante das novas tecnologias e configurações do mundo do trabalho (Arthur, \& Rousseau, 1996). Arthur (2008, p. 168, tradução nossa) escreve:

Essas carreiras, refletindo o momento em que vivemos, podem ser descritas como sendo uma resposta: (a) ao deslocamento das fronteiras ocupacionais, organizacionais, nacionais e à estruturação de novos arranjos globais de trabalho; (b) à consequente incerteza fruto da rápida geração de conhecimento e seus efeitos imprevisíveis; e (c) à maior agência individual não apenas em resposta ao deslocamento das fronteiras e à maior insegurança, mas também pela possibilidade maior de combinações de experiências de trabalho que podem ser incorporadas na carreira de alguém.
Contudo, autores como Jacoby (1999), Ricardo e Guest (2010) e Stevens (2005), sustentando-se em pesquisas de instituições como Organização de Cooperação e de Desenvolvimento Econômico (OCDE), Eurostat e U.S. Bureau of Labor Statistics, indicam discrepância entre a realidade macro do universo do trabalho e o contexto apresentado pela teoria da "carreira sem fronteiras". Para esses autores, a teoria da "carreira sem fronteiras" não é capaz de traduzir adequadamente a dinâmica da carreira contemporânea. Tão pouco a carreira estaria se tornando cada vez mais "sem fronteiras".

À primeira vista pode parecer um paradoxo que uma teoria com tamanha permeabilidade receba críticas tão incisivas. É justamente em relação a esse estranhamento que este artigo se debruçará. Buscar-se-á iluminar algumas das razões que levam o modelo teórico de "carreira sem fronteiras" a, apesar das críticas recebidas, ter a credibilidade e a permeabilidade que possui. Compreender a ambivalência da teoria permitirá contemplar maior riqueza de elementos no entendimento do fenômeno "carreira". Tal abertura mostra-se especialmente importante na medida em que se faz a transposição da teoria, desenvolvida nos Estados Unidos, para o contexto brasileiro, que possui características históricas e sociais próprias. Analisando amostra de ex-funcionários do Banco Banespa, Veloso e Dutra (2011) indicam haver discrepância entre a "carreira sem fronteiras" e a experiência de profissionais brasileiros.

Assim, para que o objetivo deste trabalho seja cumprido, serão percorridas duas etapas. Na primeira, a teoria será aprofundada ao mesmo tempo em que três categorias de críticas são apresentadas: (a) a contraposição entre velha e nova carreira; (b) a metáfora das fronteiras; e (c) a autonomia dos indivíduos. Na segunda, serão apresentados os dois conceitos centrais para a análise aqui proposta: "performatividade" (performativity) e "agentes que calculam" (calculative agents) propostos por Callon (2005) e centrais para a Actor-Network Theory (ANT). A partir desses conceitos, o aparente paradoxo da "carreira sem fronteiras" será analisado e algumas das razões para a credibilidade da teoria serão apresentadas.

Vale ressaltar que esses conceitos foram escolhidos por conferirem atenção não para teoria e realidade enquanto variáveis separadas, mas para a influência constante entre ambas por meio das ações dos atores sociais. Tal característica, própria de uma pers- 
pectiva construcionista social, possui a abrangência necessária para que a teoria seja criticada sem ser negada. Desse modo, espera-se não apenas indicar as limitações que a teoria de "carreira sem fronteiras" possui para se referir ao tema "carreira", mas também indicar a sua complexidade junto com uma perspectiva de análise replicável para outros estudos da Psicologia que tratem desse objeto.

\section{Primeira Parte}

\section{Críticas à "carreira sem fronteiras"}

Crítica à contraposição entre velha e nova carreira

Ainda que Arthur (1994, p. 297) seja cauteloso ao afirmar que as carreiras tradicionais (ou velhas) e as "sem fronteiras" (ou contemporâneas) coexistem, a frase "em linhas gerais, o cenário do emprego estável e da carreira organizacional está sumindo" deixa claro a sua crença na extinção das antigas características da carreira. A partir desse pressuposto, a oposição entre a carreira "organizacional" e a carreira "sem fronteiras", tornou-se a explicação mais simples e emblemática para esse modelo teórico (Arthur, 1994; Gunz, Evans, \& Jalland, 2000). Nesse sentido, cria-se a expectativa de que, em economias desenvolvidas, as variáveis centrais para a "carreira sem fronteiras" intensifiquem-se ao longo dos anos na medida em que as características centrais para a carreira "organizacional" percam relevância (Arthur, \& Rousseau, 1996; Defilippi, \& Arthur, 1994; Ricardo, \& Guest, 2010).

Em relação a essa previsão, duas variáveis mostram-se relevantes para avaliar o quanto essa tem se concretizado ou não: a média de tempo que os trabalhadores empregados atuam para um mesmo empregador (em inglês, job tenure) e a porcentagem de trabalhadores imigrantes. A primeira avalia o quanto as fronteiras entre empresas são superadas, a segunda, o quanto as fronteiras entre países são superadas. Vale ressaltar que os dados aqui apresentados são, em sua maior parte, gerais para trabalhadores dos diferentes países que serão referidos. Uma vez que a proposta deste trabalho é a de uma análise abrangente (tal qual propõe a "carreira sem fronteiras") optou-se por não quebrar os dados em categorias específicas de trabalhadores.

Quando se analisa o job tenure a partir de meados da década de 1990 de países considerados desenvolvidos, referências para a "carreira sem fronteiras", observa-se uma tendência de aumento na média de tempo em que os empregados em geral trabalham para seus atuais empregadores, o que contradiz a expectativa a partir da "carreira sem fronteiras". Segundo a OCDE (2017), entre 1996 e 2012 o job tenure na Alemanha aumentou de 10 para 11,5 anos, enquanto que na Espanha, passou de 9,7 para 10,9 anos. Já nos Estados Unidos, no mesmo período o job tenure aumentou de 3,8 para 4,6 anos (Hipple, \& Sok, 2013). No caso do Brasil, entre 2001 e 2013, a média aumentou de 8,4 para 9 anos (OCDE, 2017).

Estudos mais abrangentes (tanto espacialmente como temporalmente) também corroboram a falta de evidências da inexpressividade das fronteiras organizacionais. Por exemplo, Stewart (2002) identificou que o índice que contempla os pedidos de demissão e as demissões por parte do empregador (chamados de taxa de separação) variou muito pouco nos Estados Unidos entre os anos de 1976 e 2001. Kato (2001), analisando a taxa de retenção de profissionais japoneses, percebeu haver uma tendência de estabilidade desse índice, oscilando apenas em períodos de crises econômicas. Ainda, Ricardo e Guest (2010) identificaram uma tendência histórica geral de estabilidade no job tenure e no turnover na Europa, no Japão e nos Estados Unidos.

Essa tendência histórica pode ser explicada, ao menos em partes, quando se observam grupos de trabalhadores, tais como homens e mulheres. Nesse caso, observa-se que há uma diferença considerável no job tenure. Por exemplo, enquanto os homens tornaram-se mais suscetíveis a perder o emprego e, consequentemente, indicam um job tenure progressivamente menor (Boisjoly, Duncan, \& Smeeding, 1998; Farber, 2010), as mulheres evidenciam uma situação oposta (Bernhardt, Morris, Handcock, \& Scott, 1999). Para os autores, tal fenômeno seria explicado pela crescente abertura do mercado de trabalho formal às mulheres a partir da década de 1970 e pela crescente necessidade de complemento de renda nas famílias. Ricardo e Guest (2010) citam diversos estudos que mostram que, no Reino Unido, entre 1970 e 1990, a instabilidade do emprego afetou apenas os trabalhadores menos qualificados, os homens e os trabalhadores contingentes (autônomos, freelancers, consultores).

Tal diferença entre o retratado na "carreira sem fronteiras" e os dados obtidos pode ser explicada pela amostra tomada como referencial (Arthur, 1994; Arthur, \& Rousseau, 1996). Conforme Ricardo e Guest (2010) indicam, os argumentos utilizados para reforçar a validade da "carreira sem fronteiras" se referem a 
casos de indivíduos específicos ou de contextos específicos, tais como o dos profissionais de tecnologia da informação no Vale do Silício. Porém, em termos de quantidade de trabalhadores, tal contexto é pouco representativo do universo do trabalho atual: Apple, Google, Microsoft, Amazon, Intel e Cisco, juntas, empregam menos funcionários que a cadeia de supermercados Kroger (Davis, 2010). Mais ainda, o estudo de Gunz et al. (2000) indica que mesmo empresas de tecnologia, quando atuam em mercados distintos do das empresas do Vale do Silício, como a da biotecnologia, ou em outras regiões, como na Nigéria, exibem características de carreira mais próximas da organizacional do que da sem fronteiras. Com esses dados, parece haver respaldo para a observação de que o modelo de "carreira sem fronteiras" tem seu alcance limitado a determinados contextos de trabalho, como o Vale do Silício e o mercado cinematográfico dos Estados Unidos (Dany, 2008; Pringle, \& Mallon, 2003; Ricardo, \& Guest, 2010).

\section{Crítica à metáfora das fronteiras}

Ainda que Arthur e Rousseau (1996) indiquem que os aspectos objetivos não são os únicos e exclusivos aspectos com os quais o modelo de "carreira sem fronteiras" se ocupa, os aspectos ditos subjetivos (por exemplo, o desejo pela mobilidade e pela flexibilidade das relações de trabalho) não são o ponto de partida teórico nem o argumento utilizado para justificar a relevância da teoria. Esses aspectos subjetivos, de fato, passaram a ser melhor abordados somente mais adiante no tempo (Briscoe et al., 2006) como uma iniciativa de aprofundar a teoria.

Desse modo, nos últimos anos, a ideia de fronteira subjetiva ganhou força no interior da "carreira sem fronteiras” trazendo à tona, então, não apenas o concreto rompimento das fronteiras, mas também o desejo ou a intenção de fazê-lo (Sullivan, \& Arthur, 2006). Contudo, como o próprio instrumento desenvolvido a partir dessa ideia indica (Briscoe et al., 2006), a não ser que exista completa imobilidade física da carreira e nenhuma intenção de mudança, a carreira de alguém será classificada como potencialmente "sem fronteiras" (Arnold, \& Cohen, 2008). Outra objeção pode ser levantada seguindo-se a hipótese relativa à migração e à imigração: será que, em geral, o desejo por mobilidade aumentou ou apenas temos um mesmo desejo por mobilidade que possui maior alcance?
Desde 2004, a participação de trabalhadores imigrantes aumentou em diversos países (OCDE, 2017), indicando que as fronteiras entre países são, sim, transpostas com maior facilidade e em uma intensidade cada vez maior. Curiosamente, enquanto isso, a taxa de migração interna para diversos países, como os Estados Unidos, tem decrescido ao longo dos últimos 30 anos (Molloy, Smith, \& Wozniak, 2011). Esse fato permite levantar a hipótese que, por consequência do desenvolvimento dos meios de transporte, aquelas pessoas que se moviam dentro de um mesmo país passaram a transitar em uma escala global. Nesse sentido, o desejo por mobilidade não teria mudado em intensidade, mas em alcance.

Além disso, o modelo de "carreira sem fronteiras" pouco considera que as pessoas podem perceber as fronteiras subjetivas e objetivas como tendo pesos e relevâncias diferentes. Como Inkson (2006) exemplifica, existem diferentes níveis de fronteiras objetivas, como a organizacional, a do ramo da indústria e a da profissão, que podem ser vistas de formas muito distintas de acordo com a dinâmica de interações estabelecidas, ou seja, a força das fronteiras não estaria no indivíduo ou no contexto. Por exemplo, por mais que deseje atravessar uma fronteira, uma pessoa pode desistir de uma mudança por receber a notícia de que terá um filho, por ter um chefe persuasivo que lhe oferece um aumento, por ter lido no jornal que o momento é de crise econômica ou por ter seu visto negado devido ao conflito político entre dois países.

Outro aspecto largamente questionado por diversos acadêmicos (Arnold, \& Cohen, 2008; Gunz et al., 2000; Inkson, 2006) é quanto à capacidade da metáfora "sem fronteiras" traduzir uma nova realidade do trabalho. Tanto a carreira organizacional como a sem fronteiras enfatizam as fronteiras, sejam elas objetivas ou subjetivas (Arthur, 1994; Defillippi, \& Arthur, 1994). Nesse sentido, críticas se acumulam em referência à falta de capacidade que a metáfora "sem fronteiras" tem de apresentar um cenário distinto em que as fronteiras já não possuem a mesma relevância. Conforme Gunz et al. (2000) apontam, para que a relevância da "carreira sem fronteiras" seja analisada, é necessário que as fronteiras continuem a existir do mesmo modo que antigamente. Só assim poder-se-á observar se as fronteiras são atravessadas ou não.

Além disso, estudos como o de Jackson, Goldthorpe e Mills (2005) apresentam as diversas fronteiras que ainda hoje delimitam as carreiras profissionais, 
tais como o emprego prévio e o nível de escolaridade. Ainda, o rompimento de fronteiras faz parte inclusive do modelo de carreira organizacional (Arnold, \& Cohen, 2008). Por exemplo, em estudo que acompanhou profissionais entre os anos 1957 e 1972, Topel e Ward (1992) observaram que o trabalhador americano atuou em média para 10 diferentes empregadores durante a carreira. Já Hashimoto e Raisian (1985) identificaram que em média o trabalhador japonês de 65 anos teria trabalhado para cinco empregadores diferentes em 1977.

Ainda mais, Gunz et al. (2000), Inkson et al. (2012) e Saxenian (1996) sugerem que, se a "carreira sem fronteiras" se aplica a contextos específicos e não ao mercado de trabalho como um todo, esse fato se configuraria como uma fronteira que ofereceria resistência a quem deseje ingressar em ou sair de, por exemplo, empresas do Vale do Silício. Desse modo, a conclusão de Inkson et al. (2012) parece pertinente: a experiência de trabalho contemporânea não indica uma ausência de fronteiras, mas sim uma mudança na forma com que as diversas fronteiras são atravessadas. Tal cenário pede justamente uma atenção especial ao estudo das fronteiras e não o inverso.

\section{Crítica à ênfase na autonomia do indivíduo}

Outro aspecto muito criticado da "carreira sem fronteiras" é a ênfase que os estudiosos desse modelo teórico conferem às ações individuais (Inkson, 2006; Inkson et al., 2012; Ricardo, \& Guest, 2010). A preponderância das ações individuais, antes de ser um fundamento para a teoria, é uma consequência lógica para a crença na perda de força das organizações. Na medida em que as organizações deixam de ser capazes de oferecer a segurança da progressão da carreira ou mesmo de oferecer um caminho nítido de progressão, os interesses individuais e as decisões pessoais tornam-se centrais na construção da carreira (Bendassolli, 2009).

Assim, as carreiras passam a ser avaliadas como uma construção independente, individualmente almejada e avaliada pelo indivíduo (Arthur, \&Hall, 1989). Não é à toa que temas como valores pessoais (Mirvis, \& Hall, 1994), sucesso subjetivo (Arthur, Khapova \&Wilderom, 2005), empreendedorismo (Borges, 2012) e o pedido de demissão (Mainero, \& Sullivan, 2005) ganham grande relevância em meio à "carreira sem fronteiras". Ainda, além de ser uma consequência da nova composição do trabalho, a individualidade na construção da carreira ganhou valor positivo enquanto emancipação das tra- dicionais e ultrapassadas amarras do trabalho (Hall, \& Moss, 1998; Ricardo, \& Guest, 2010).

Contudo, evidências sugerem que as pessoas estão menos no controle do que é indicado pela teoria. Nos Estados Unidos, a porcentagem dos desligamentos atribuídos à decisão do trabalhador caiu no intervalo entre 2000 e 2013 , indo de $57,5 \%$ do total de desligamentos para $54,5 \%$, atingindo a menor expressividade em 2009, com 41,9\% (U.S. Bureau of Labor Statistics, 2014). Esse dado indica, de um modo geral, um maior interesse por parte dos empregados em se manter no mesmo trabalho, e, ao mesmo tempo, uma maior participação das empresas nos rumos da carreira dos (ex) empregados.

Além disso, dados sobre a quantidade de trabalhadores autônomos, freelancers e consultores nos Estados Unidos, indicam a redução desse grupo de profissionais que simbolizam o desprendimento da "carreira organizacional". Em 1995, eles representavam $6,34 \%$ dos profissionais; em 2005, 4,11\% (U.S. Bureau of Labor Statistics, 2014). Já na Alemanha, observa-se uma leve queda na porcentagem de trabalhadores autônomos entre os anos 2004 e 2012, indo de $10,54 \%$ para $10,14 \%$ (Eurostat, 2014).

Nesse sentido, pode-se observar que diversas variáveis mostram uma redução da autonomia dos indivíduos em relação aos seus empregadores, o que indica não apenas a possibilidade de uma indesejada dependência para alguns grupos de trabalhadores, mas também de certo nível de dependência desejado diante das inseguranças do universo do trabalho. Uma relação não fortuita parece haver entre os pedidos de desligamento e o índice de emprego. Talvez por sentirem-se confiantes de que poderão retornar ao mercado de trabalho, os trabalhadores participam mais ativamente nos desligamentos em períodos com alto índice de emprego. Por outro lado, a situação se inverte diante de queda nos índices de emprego, como logo após a crise de 2008.

Assim, conforme já citado, as proposições do modelo de "carreira sem fronteiras" não são amplamente verificáveis como a teoria propõe, restringindo-se a grupos específicos de trabalhadores (Pringle, \& Mallon, 2003). Ainda, chama a atenção o contraste entre a abrangência da definição de carreira, na qual a "carreira sem fronteiras" se baseia e a atenção demasiada conferida a grupos específicos de trabalhadores, como os executivos de empresas de tecnologia dos Estados Unidos (Guest, \& Sturges, 2007). 


\section{Segunda Parte}

\section{Performatividade, os agentes que calculam e a relevância da "carreira sem fronteiras"}

Para analisar o aparente paradoxo entre a grande permeabilidade da "carreira sem fronteiras" e a baixa correspondência com índices macrossociais serão utilizados dois conceitos apresentados por Callon (2005, 2006), "performatividade" e "agentes que calculam". Ambos os conceitos são emblemáticos da ANT, que se nutre da perspectiva construcionista social. Esta, por sua vez, oferece a abrangência necessária para que se investigue o problema proposto de modo a contemplar seu aparente paradoxo sem ter de negá-lo. Ainda, tal perspectiva serve de meio para que se possa transpor teorias, como a 'carreira sem fronteiras', de modo crítico entre diferentes contextos.

Como Callon (2006) relata, central para sua teoria é a pergunta: "como uma teoria pode estar fora da realidade que descreve e, ao mesmo tempo, participar na construção dessa realidade enquanto objeto?". É justamente para essa pergunta que o conceito de performatividade oferece uma resposta. Em termos gerais, esse conceito indica que teoria e realidade não são separáveis, uma vez que um influencia o outro seja validando-o ou reconstruindo-o.

Um dos pressupostos teóricos que sustentam a performatividade é o que o filósofo Goodman (1984) expôs quanto às regras e formas do mundo; para ele, essas não estão disponíveis para serem descobertas, mas sim, são impostas pelos próprios homens de acordo com suas interpretações do mundo. Assim, para que a performatividade faça sentido, a realidade não pode ser entendida como um campo em que a verdade está disponível para ser conhecida. A verdade, e, consequentemente, a realidade, devem ser compreendidas como objetos constantemente construídos. Ainda segundo Goodman (1984), as teorias são importantes não porque são verdades, mas porque ajudam os seres humanos a lidar com aquilo que entendem como realidade. Uma vez que não relaciona teoria com verdade, a performatividade permite analisar a "carreira sem fronteiras" para além do aparente paradoxo apontado no início deste artigo.

Quando Callon (2005) se refere a essa necessidade de lidar com o mundo com a qual as teorias auxiliam, ele se refere à necessidade que o homem tem de calcular. O cálculo, ou seja, a capacidade de planejar ações é um requisito para que a interação social seja orga- nizada e possível. Considerar um contexto que careça dessa organização, não permitindo previsibilidade e compreensão mínima dos acontecimentos, implica em abandoná-lo como campo social. Sem a possibilidade do cálculo, cada sujeito agiria como bem entendesse, impossibilitando que certa arena fosse delimitada; arena que, para o caso da "carreira sem fronteiras", pode ser entendida como o universo do trabalho.

Os cálculos, por sua vez, para serem feitos, exigem que os agentes utilizem certas ferramentas para interpretar as informações disponíveis. As teorias, nesse sentido, funcionariam como um "manual de instruções” (Callon, 2006). Ainda, dependendo da ferramenta (teoria) que se tem em mãos, a análise do contexto será distinta e levará a ações diferentes. Consequentemente, ao agir, o sujeito interferirá no próprio cenário que analisou e o modificará, influenciando futuras teorias e ferramentas. Essa capacidade de a teoria intervir na realidade e moldá-la é o que Callon (2005) chama de performatividade da teoria.

Dentro dessa análise, uma teoria não é avaliada quanto à sua proximidade da verdade, mas ao seu sucesso ou fracasso em auxiliar as pessoas nos seus cálculos e nas suas atribuições de sentido à realidade (Quine, 1981). Isso coloca as teorias dentro de uma complexa rede de influências, aceitações e credibilidade dos atores envolvidos nas enunciações das teorias que transcendem o conteúdo em si (Callon, 2005). Nesse conjunto de fatores, a validação empírica, que não é tão ampla quanto se esperaria no caso da "carreira sem fronteiras", é apenas um dentre inúmeros fatores que interferem no sucesso de uma teoria.

\section{Análise da "carreira sem fronteiras" e sua performatividade}

Assim, à luz da performatividade, temos um cenário em que o modelo de "carreira sem fronteiras" possui grande permeabilidade, sobretudo nos meios acadêmico e empresarial, ao mesmo tempo em que indicadores macrossociais evidenciam um distanciamento entre teoria e mercado de trabalho.

Dentre as inúmeras formas de analisar o sucesso da "carreira sem fronteiras", este será analisado por duas principais perspectivas: (a) em um nível teórico, referindo-se ao cálculo dos agentes e (b) em um nível de interacional, referindo-se à credibilidade de certos agentes envolvidos no universo do trabalho. 
"Carreira sem fronteiras" e o cálculo dos agentes

Nos artigos em que criticam a "carreira sem fronteiras", Ricardo e Guest (2010) e Inkson et al. (2012) levantam a hipótese de que um dos fatores cruciais para o sucesso desse modelo teórico é a retórica consonante com o discurso da globalização. De fato, o começo da década de 1990, período em que a "carreira sem fronteiras" foi apresentada, é símbolo do fortalecimento das novas tecnologias no contexto do trabalho, sobretudo com a disseminação da internet, e do rompimento de fronteiras na transmissão de informações.

Assim, o processo de mudança que teve início por volta da década de 1970 (Davis, 2010) foi se tornar mais evidente na década de 1990, período do lançamento da "carreira sem fronteiras". Esse é, de fato, um dos argumentos centrais de Arthur (1994, p. 296, tradução nossa): "Em que medida os modelos de emprego norteados por arranjos temporários e pelo mercado têm capturado nosso interesse? Será que consideramos aquilo que as carreiras, enquanto campo do aprendizado individual, podem significar para o aprendizado organizacional?". O discurso da "carreira sem fronteiras" ofereceu, em relação ao campo de estudo da carreira, uma proposta de atualização que parecia necessária frente às intensas mudanças no mundo do trabalho.

Consequentemente, a "carreira sem fronteiras" ofereceu uma nova ferramenta para os cálculos das pessoas. Ricardo e Guest (2010) propõe que central para o sucesso da "carreira sem fronteiras" foi e ainda é a simplificação (muitas vezes demasiada) dos padrões de carreira. Ainda que muitos elementos sejam deixados de lado, a simplificação é bem-vinda diante de um contexto de extrema complexidade e aparente ilegibilidade (Callon, 2005), pois torna o cálculo e a atribuição de sentido possíveis. Além disso, conforme sugerido em trabalho prévio (Sewaybricker, 2012), o processo de simplificação teórica favorece a sua repetição e o seu fortalecimento fora do meio acadêmico, como, por exemplo, nas organizações.

Para a simplificação promovida pela "carreira sem fronteiras" há um elemento central: a ênfase no indivíduo. A importância da autonomia do indivíduo é especialmente grande para a teoria, pois é ela que permite aos sujeitos a segurança dos cálculos em meio ao contexto conturbado. Sendo incapaz de antever com demissões em massa, crises econômicas, expatriações, compras e fusões, não seria promissor para o indivíduo respaldar seus planos profissionais em expectativas sobre esse contexto. Mais oportuno se torna respaldar os planos em aspectos pessoais como valores pessoais e desejos (Savickas et al., 2009).

Retornando ao passado recente, mais propriamente a 1994, ano da publicação do artigo inaugural da "carreira sem fronteiras", tem-se outro fator além do retórico que favoreceu a credibilidade desse modelo teórico. Nos Estados Unidos, o período entre os anos 1992 e 2000 foi marcado por uma queda contínua do desemprego, indo de 7,5\% para 3,95\% (U.S. Bureau of Labor Statistics, 2014). Quando se compara o índice de desemprego com outras variáveis de grande importância para a validação da "carreira sem fronteiras", verifica-se que o primeiro possui moderada correlação com, por exemplo, os pedidos de demissão e o job tenure. Pode-se intuir, assim, que em um cenário com maior segurança de emprego, mais pessoas se arriscarão a deixar seus trabalhos em busca de melhores oportunidades, seja abrir negócio próprio ou buscar emprego em outra empresa que pague melhor. Assim, a inauguração da "carreira sem fronteiras" foi marcada por um período em que as próprias evidências empíricas dos Estados Unidos foram, por alguns anos, mais favoráveis à teoria.

Outra possível sustentação para a relevância da "carreira sem fronteiras" está na percepção subjetiva da falta de fronteiras ou do constante rompimento dessas. Se anteriormente, quando as fronteiras eram rompidas, mudava-se para a empresa vizinha ou para a cidade próxima. Com o acesso maior à informação e aos meios de transporte de longa distância tornou-se mais fácil mudar para empresas distantes e, inclusive, para outros países. O impacto para a percepção de se estar "sem fronteiras" é muito maior quando se muda para um país a muitas horas de avião do que quando se muda para uma cidade próxima, onde os valores e a cultura provavelmente serão mais semelhantes.

Essa análise corrobora as interpretações de sociólogos como Bauman (2003) e Sennett (2002) que apresentam a perda da representatividade dos espaços físicos para a noção de comunidade, no caso de Bauman, e identidade, no caso de Sennett. Para ambos, há na atualidade uma experiência muito maior de falta de fronteiras mesmo quando não há mobilidade física envolvida. Isso equivaleria a dizer que, mesmo sem se atravessar fronteiras concretas, há a sensação de que elas não possuem a mesma força que antigamente. Por exemplo, ainda que certas pessoas queiram a segurança do emprego e não desejem a mobilidade, a tecnologia as coloca em contato com informações e 
culturas que invadem suas fronteiras e enevoam suas identidades, intensificando a sensação de não pertencerem a nenhum lugar. Desse modo, mesmo que a "carreira sem fronteiras" não seja tenha suporte empírico tão amplo, as proposições teóricas soariam como plausíveis, conquistando credibilidade.

"Carreira sem fronteiras" e a interação dos agentes

Além do papel da teoria em si, os agentes que divulgam e reproduzem essa teoria também têm papel determinante para o seu sucesso. Variáveis como a reputação de quem fala e da instituição de onde se fala, bem como a importância dos locais onde essa teoria passa a ser reproduzida interferem decisivamente para o sucesso ou fracasso dela (Callon, \& Latour, 1981).

No caso da "carreira sem fronteiras", deve-se primeiro destacar a importância das universidades e escolas de negócios dos Estados Unidos que tradicionalmente figuram entre as melhores do mundo segundo diversos rankings, como o da Times Higher Education e da QS World Universities Rankings. Esses dois rankings deixam claro a relevância das instituições de educação desse país para os âmbitos acadêmico e profissional globais.

Embora a Suffolk University e seu braço de negócios, a Sawyer Business School, para a qual Michael Arthur trabalha, figurem apenas ao redor da $100^{\text {a }}$ posição nos dois rankings mencionados, elas não fogem da regra de ser consideradas referência no nível global e ter grande número de estudantes de outros países, sendo um legítimo ambiente "sem fronteiras".

Quanto aos locais onde esse específico modelo teórico passa a ser reproduzido, deve-se destacar a importância que as grandes empresas têm para o sucesso dessa teoria (Inkson et al., 2012). Essas teriam um duplo interesse na reprodução da "carreira sem fronteiras", pela justificativa da flexibilização das operações e pela valorização da agência dos trabalhadores.

Frente às necessidades de flexibilização das empresas, a "carreira sem fronteiras" pode ser entendida como um poderoso instrumento legitimador. Cohen e Mallon (1999) identificam o potencial da "carreira sem fronteiras" para justificar práticas de demissões, downsizing e reduções de benefícios. Na medida em que o indivíduo constituinte da "carreira sem fronteiras" é autônomo e nada deve esperar da organização, ele não deve culpá-la em caso de quebra do vínculo de emprego. De fato, os próprios vínculos já não devem mais ser encarados da mesma forma pelo indivíduo, como a frase de Jack Welch, ex-CEO da GM, indica: "Você pode dar empregabilidade para uma vida inteira ao oferecer treinamento, ao tornar as pessoas adaptáveis e aptas a irem a outros lugares executar tarefas diferentes. Mas você não pode garantir emprego para uma vida inteira" (Davis, 2010, p. 31, tradução nossa). Para essa linha de pensamento, o indivíduo deve se preocupar com seus referenciais internos, sua rede e suas habilidades e a empresa deve pensar livremente na flexibilização de suas operações.

Ao mesmo tempo em que justifica certas ações das organizações, o discurso da "carreira sem fronteiras" fortalece a imagem do trabalhador em sua potência para controlar os rumos da carreira. Essa dupla função da teoria operaria na mesma lógica daquilo que diversos autores (Bendassolli, 2007; Ehrenberg, 2010) identificam como discurso contemporâneo da gestão de pessoas, sendo a principal função desse discurso a de reduzir tensões entre indivíduo e organização do trabalho.

Sendo a própria organização do trabalho beneficiada pela "carreira sem fronteiras", pode-se identificar também o quanto suas práticas internas, sobretudo da área de gestão de pessoas, são relevantes para a performatividade da teoria. Por exemplo, uma área de desenvolvimento que adote a "carreira sem fronteiras" como referencial teórico reproduzirá a "carreira sem fronteiras" nos projetos de encarreiramento, incentivando que os profissionais tenham experiências em áreas diversas da organização (como as chamadas movimentações laterais e as expatriações). Já uma área de recrutamento reproduzirá a teoria tanto na avaliação de pessoas, entendendo que a experiência em múltiplas funções/organizações é mais positiva do que em uma única posição/organização, como na maior abertura dos processos seletivos para profissionais de fora da organização. Nesses exemplos, a teoria torna-se performativa da experiência de trabalho sem que haja, necessariamente, uma prévia correspondência com a realidade macrossocial. Nesse caso, a própria teoria passa a criar a realidade do mercado de trabalho sobre a qual se refere.

\section{Conclusão}

Diante do conjunto das análises aqui apresentadas, o paradoxo da "carreira sem fronteiras" não mais se apresenta como, de fato, um paradoxo uma vez que a teoria não necessita somente de amplas evidências empíricas para ser validada. Desse modo, partindo 
dos conceitos de performatividade e cálculo dos agentes, identificou-se seis fatores que influenciam positivamente na reputação da "carreira sem fronteiras".

(1) A simplificação que a teoria faz do contexto profissional, sobretudo por meio da ênfase no indivíduo, oferece um cálculo possível de ser realizado no imprevisível cenário globalizado. (2) Além disso, empiricamente, a teoria teve crescente respaldo durante a década em que foi lançada, o que provavelmente favoreceu a percepção de sua validade. (3) Ainda, a teoria evidencia consonância com o que sociólogos como Bauman indicam ser típico da contemporaneidade: a percepção de que as fronteiras são rompidas a todo instante e mesmo contra a vontade das pessoas.

Já em relação à carreira profissional, no âmbito acadêmico americano e as grandes empresas mostram-se especialmente importantes para reprodução e validação da "carreira sem fronteiras". (4) As universidades e as escolas de negócio americanas, como a Suffolk University e seu braço de negócios, a Sawyer Business School, onde o fundador da "carreira sem fronteiras" leciona, são amplamente reconhecidas pela sua credibilidade e por serem um espaço de convívio entre pessoas de diferentes nacionalidades. (5) Além disso, há consonância entre o papel da "carreira sem fronteiras" e o que teóricos apresentam serem os argumentos do discurso gerencialista intrínsecos à flexibilização das empresas. Esse modelo de carreira acaba por justificar demissões, reduções e mudanças organizacionais, uma vez que não se pode esperar a fidelidade do vínculo empregatício. Ao mesmo tempo, a teoria serve aos profissionais, que, inseguros diante do novo cenário, são "empoderados" a se apropriar e dar sentido para suas carreiras (ao menos verbalmente). (6) Por fim, o conhecimento disseminado pela "carreira sem fronteiras" serve de subsídio para práticas da área de gestão de pessoas das organizações, que tenderá a valorizar/reforçar aqueles profissionais com carreira "sem fronteiras" e se empenhará para que a própria estrutura interna potencialize experiências "sem fronteiras".

Ao longo deste artigo, pode-se verificar que as críticas tecidas ao modelo de "carreira sem fronteiras" são consistentes e encontram relevante sustentação empírica. Ainda assim, a teoria, embora limitada em retratar um aspecto geral do mercado de trabalho, é bem-sucedida em auxiliar os indivíduos a calcularem e é respaldada por importantes agentes. Todavia, espera-se que tenha ficado patente a complexidade do universo do trabalho contemporâneo, sobretudo em relação à mudança da qualidade (e não dissolução) das diferentes fronteiras.

O método aqui esboçado, de análise da "carreira sem fronteiras" segundo a lente da performatividade, pode ser utilizado de forma ainda mais profunda e sistemática. Aqui buscou-se, antes, trazer à tona parte da dinâmica entre atores que constroem, reproduzem e validam a teoria de carreira, dinâmica esta que pode acrescentar novas nuances e enriquecer o debate referente ao tema no campo da Psicologia.

\section{Referências}

Arnold, J., \& Cohen, L. (2008). The psychology of career in industrial and organizational setting: A critical but appreciative analysis. In G. P. Hodgkinson, \& J. K. Ford (Ed.), International review of industrial and organizational psychology (pp. 1-44). New York, NY: Wiley.

Arthur, M. B. (1994) The boundaryless career: A new perspective for organization inquiry. Journal of Organizational Behavior, 15, 295-306. https:// doi.org/10.1002/job.4030150402

Arthur, M. B. (2008). Examining contemporary careers: a call for interdisciplinary inquiry. Human Relations, 61(2), 163-186. https://doi.org/10.1177/0018726707087783

Arthur, M. B., \& Hall, D. T. (1989). Handbook of career theory. New York, NY: Cambridge University Press.

Arthur, M. B., Khapova, S. N., \& Wilderom, C. P. (2005). Career success in a boundaryless career world. Journal of Organizational Behaviour, 26(2), 177-202. https://doi.org/10.1002/job.290

Arthur, M. B., \& Rousseau, D. M. (1996). The boundaryless career a new employmet principle for a new organizational era. New York, NY: Oxford University Press.

Bauman, Z. (2003). Comunidade: A busca por segurança no mundo atual. Rio de Janeiro, RJ: Jorge Zahar.

Bendassolli, P. F. (2007). Trabalho e identidade em tempos sombrios. São Paulo, SP: Ideias e Letras. 
Bendassolli, P. F. (2009). Recomposição da relação sujeito-trabalho nos modelos emergentes de carreira. Revista de Administração de Empresas, 49(4), 387-400. https://doi.org/10.1590/S0034-75902009000400003

Bernhardt, A., Morris, M., Handcock, M. S., \& Scott, M. (1999). Trends in job instability and wages for young adult men. Journal of Labor Economics, 17(S4), S65-90. https://doi.org/10.1086/209943

Boisjoly, J., Duncan, G. J., \& Smeeding, T. (1998). The shifting incidence of involuntary job losses from 1968 to 1992. Industrial Relations, 37(2), 207-231. https://doi.org/10.1111/0019-8676.00083

Borges, J. F. (2012). Quando o empreendedorismo gera o empreendedor: as microfundações da carreira empreendedora (Tese de doutorado), Programa de Pós-graduação em Administração de Empresas, Universidade de São Paulo, São Paulo, SP.

Briscoe, J. P., Hall, D. T., \& Frautschy Demuth, R. L. (2006). Protean and boundaryless careers: an empirical exploration. Journal of Vocational Behavior, 69(1), 30-47. https://doi.org/10.1016/j.jvb.2005.09.003

Callon, M. (2005). The laws of the market. London: Blackwell.

Callon, M. (2006). What does it mean to say that economics is performative? In D. Mackenzie, F. Muniesa, \& L. Siu (Ed.), Do economists make markets? On the performativity of economics. Princeton, NJ: Princeton University Press.

Callon, M., \& Latour, B. (1981). Unscrewing the big leviathan: Or how actors macrostructure reality, and how sociologists help them to do so? In K. Knorr, \& C. Cicourel (Ed.), Advances in social theory and methodology (277-303). London: Routledge and Kegan Paul.

Cohen, L., \& Mallon, M. (1999). The transition from organizational employment to portfolio working: Perceptions of boundarylessness. Work, Employment and Society, 13(2), 329-352.

Dany, F. (2008). 'Free actors' and organizations: Critical remarks about the new career literature, based on French insights. The international Journal of Human Resources Management, 14(5), 821-838. https://doi. org/10.1080/0958519032000080820

Davis, G. F. (2010). After the Ownership Society: Another world is possible. In N Lounsbury, P. M. Hirsch (Eds.), Markets on trial: The economic sociology of the U. S. financial crisis (pp. 331-356, Research in the Sociology of Organizations, Vol. 30B). Bingley: Emerald Group.

Defillippi, R. J., \& Arthur, M. B. (1994). Ther boundaryless career: A competency-based perspective. Journal of Organizational Behavior, 15(4), 307-324. https://doi.org/10.1002/job.4030150403

Eby, L. T., Butts, M., \& Lockwood, A. (2003). Predictors of success in the era of the boundaryless career. Journal of Organizational Behavior, 24(6), 689-708. https://doi.org/10.1002/job.214

Ehrenberg, A. (2010). O culto da performance. São Paulo, SP: Ideias e Letras.

Eurostat (2014). Database. Recuperado de http://ec.europa.eu/eurostat/data/database

Farber, H. S. (2010). Job loss and the decline in job security in the United States. In: K. Abraham, J. R. Spletzer, \& M. Harper, Labor in the new economy (pp. 223-262). Chicago, Ill: University of Chicago Press.

Goodman, N. (1984). Of mind and other matters. Cambridge: Harvard University Press.

Guest, D. E., \& Sturges, J. (2007). Living to work: Working to live: Conceptualisations of careers among contemporary workers. In H. Gunz, \& M. Peiperl (Ed.), Handbook of career studies (pp. 310-326). London: Sage.

Gunz, H., Evans, M., \& Jalland, M. (2000). Career boundaries in a 'Boundaryless' world. In M. Peiperl, M. B. Arthur, R. Gofee, \&T. Morris (Ed.), Career frontiers: New conceptions of working lives (pp. 24-53). Oxford: Oxford University Press.

Hall, D. T. (2002). Careers in and out of organizations. Thousand Oaks: Sage.

Hall, D. T., \& Moss, J. E. (1998). The new protean career contract: Helping organizations and employees adapt. Organizational Dynamics, 26(3), 22-37. https://doi.org/10.1016/S0090-2616(98)90012-2

Hashimoto, M., \& Raisian, J. (1985). Employment tenure and earnings profiles in Japan and the United States. The American Economic Review, 75(4), 721-735. Recuperado de https://www.jstor.org/stable/1821350?seq=1\#page scan_tab_contents

Higgins, M. C., Dobrow, S. R. \& Roloff, K. S. (2010). Optimism and the boundaryless career: The role of developmental relationships. Journal of Organizational Behavior, 31(5), 749-769. https://doi.org/10.1002/job.693 
Hipple, S. F. \& Sok, E. (2013). Tenure of American Workers. U.S. Bureau of Labor Statistics. Recuperado de http:// www.bls.gov/spotlight/2013/tenure/pdf/tenure.pdf

Inkson, K. (2006). Protean and boundaryless careers as metaphors. Journal of Vocational Behavior, 69(1), 48-63. https://doi.org/10.1016/j.jvb.2005.09.004

Inkson, K., Gunz, H., Ganesh, S. \& Roper, J. (2012). Boundaryless career: Bringing back boundaries. Organization Studies, 33(3), 323-340. https://doi.org/10.1177/0170840611435600

Jackson, M., Goldthorpe, J. H., \& Mills, C. (2005). Education, employers and class mobility. Research in Social Stratification and Mobility, 23, 3-33.

Jacoby, S. M. (1999). Are career jobs headed for extinction? California Management Review, 42, 123-145. https://doi. org/10.1016/S0276-5624(05)23001-9

Kato, T. (2001). The end of lifetime employement in Japan? Evidence from national surveys and field research. Journal of the Japanese and International Economies, 15, 489-514.

Mainiero, L. A., \& Sullivan, S. E. (2005). Kaleidoscope Careers: An alternative exploration for the "opt-out" revolution. Academy of Management Executive,19(1|), 110-123. Recuperado de http://www.jstor.org/stable/4166156

Malvezzi, S. (1999). Empregabilidade e carreira. Cadernos de Psicologia Social do Trabalho,2, 64-68. Recuperado de http://www.revistas.usp.br/cpst/article/view/25824/27556

Mirvis, P. H., \& Hall, D. T. (1994). Psychological success and the boundaryless career. Journal of Organizational Behavior, 15(4 spe), 365-380. Recuperado de http://www.jstor.org/stable/2488432

Molloy, R., Smith, C. L., \& Wozniak, A. (07 de Agosto de 2013). Declining migration within the U.S.: The role of the labor market. Washington, DC: Federal Reserve Board. Recuperado de www.federalreserve.gov/pubs/ feds/2013/201327/201327pap.pdf

Organization for Economic Co-operaion and Development - OCDE. (2017). Welcom to OECD Stat. Paris: o autor. Recuperado de http://stats.oecd.org/

Pringle, J. L., \& Mallon, M. (2003). Challenges for the boundaryless career odyssey. International Journal of Human Resources Management,14(5), 839-853. https:/ / doi.org/10.1080/0958519032000080839

Quine, W. (1981). Theories and things. Cambridge: Harvard University Press.

Ricardo, A. R., \& Guest, D. (2010). Have careers become boundaryless? Human Relations,63(8), 1157-1175. https:// doi.org/10.1177/0018726709354344

Savickas, M. L., Nota, L., Rossier, J., Dauwalder, J-P., Duarte, M. E., Guichard, J., Soresi, S. et al. (2009). Life designing: A paradigm for career construction in the 21st century. Journal of Vocational Behavior, 75(3), 239-250. https:// doi.org/10.1016/j.jvb.2009.04.004

Saxenian, A. (1996). Beyond boundaries: Open labor markets and learning in Silicon Valley. In M. B. Arthur, \& D. M. Rousseau (Eds.), The boundaryless career: A new employment principle for a new organizational era (pp. 23-39). Oxford: Oxford University Press.

Sennett, R. (2002). A corrosão do caráter. Rio de Janeiro,RJ: Record.

Sewaybricker, L. E. (2012). A felicidade na sociedade contemporânea (Dissertação de mestrado). Programa de Pós-graduação em Psicologia Social, Universidade de São Paulo Paulo, São Paulo, SP.

Stevens, A. H. (2005). The more things change, the more they remain the same: Trends in long-term employment in the United States, 1969-2002 (Working paper, Vol. 11878). Cambridge, MA: National Bureau of Economic Research.

Stewart, J. (7 de Agosto de 2002). Recent trends in job stability and job security: Evidence from the March CPS. Washington, DC: U.S. Bureau of Labor Statistics; 2002. Disponível em: https://www.bls.gov/ore/pdf/ec020050.pdf

Sullivan, S. E., \& Arthur, M. B. (2006). The evolution of the boundaryless career concept: Examining physical and psychological mobility. Journal of Vocational Behavior, 69(1), 19-29. https://doi.org/10.1016/j.jvb.2005.09.001

Topel, R. H., \&Ward, M. P. (1992). Job mobility and the careers of young men. Quarterly Journal of Economics, 107(2), 439-479. Recuperado de http://www.jstor.org/stable/2118478

U.S. Bureau of Labor Statistics (2014). Job openings and labor turnover survey . Recuperado de http://www.bls.gov/ jlt/data.ht 
Veloso, E. F. R., \& Dutra, J. S. (2011). Carreiras sem fronteiras na gestão pessoal da transição profissional: Um estudo com ex-funcionários de uma instituição privatizada. Revista de Administração Contemporânea, $15(5), 834-854$. http://dx.doi.org/10.1590/S1415-65552011000500004

\section{Luciano Espósito Sewaybricker}

Doutor pelo do Departamento de Psicologia Social do Instituto de Psicologia da Universidade de São Paulo (USP), São Paulo - SP. Brasil. Professor de Psicologia Social no Centro Universitário São Camilo, São Paulo - SP. Brasil. E-mail: luciano.sewaybricker@gmail.com

Endereço para envio de correspondência:

Rua Cunha Gago, 235, sala 24. Pinheiros. CEP: 05421-000. São Paulo - SP. Brasil.

Recebido 15/12/2016

Reformulado 11/08/2017

Aprovado 20/10/2017

Received 12/15/2016

Reformulated 08/11/2017

Approved 10/20/2017

Recibido 15/12/2016

Reformulado 11/08/2017

Aceptado 20/10/2017

Como citar: Sewaybricker, L. E. (2018). Carreira sem fronteiras: Limite e aplicabilidade de uma teoria clássica. Psicologia: Ciência e Profissão, 38(1), 129-141. https://doi.org/10.1590/1982-3703004532016

How to cite: Sewaybricker, L. E. (2018). Boundaryless career: Limitations and applicability of a classical theory. Psicologia: Ciência e Profissão, 38(1), 129-141. https://doi.org/10.1590/1982-3703004532016

Cómo citar: Sewaybricker, L. E. (2018). Carrera sin fronteras: Límites y aplicabilidad de una teoría clásica. Psicologia: Ciência e Profissão, 38(1), 129-141. https://doi.org/10.1590/1982-3703004532016 